\title{
Interaction of Sodium Ions with Cationic Surfactant Interfaces
}

\section{Sergio Murgia, ${ }^{[a]}$ Filippo Portesani, ${ }^{[a]}$ Barry W. Ninham, ${ }^{[b]}$ and Maura Monduzzi*[a]}

\begin{abstract}
The thermodynamically stable microemulsion and lamellar phases in the didodecyldimethylammonium bromide/water/ $n$-decane ternary system were explored in the presence of $\mathrm{NaBr}$ to gain information on sodium ion-interface interactions. Experimental results, obtained by different NMR
\end{abstract}

techniques, strongly suggest accumulation of sodium ions at the cationic interface. This apparently counterintui-

Keywords: interfaces $\cdot$ NMR
spectroscopy $\cdot$ phase transitions
sodium $\cdot$ surfactants

tive result is explained by invoking the dispersion potential experienced by the ions near the interface. A mechanism is proposed that can account for the dramatic shrinkage of the microemulsion phase region when an electrolyte is added.

\section{Introduction}

Background: The stability of colloidal systems is determined by a delicate interplay of various intermolecular forces. Supramolecular microstructures, long-range ordered liquid crystals, and also some simple biological systems are often used as models that reflect the role of different intermolecular forces in stabilization processes and in the evolution of microstructure. For more than half a century the DerjaguinLandau-Verwey-Overbeek (DLVO) theory ${ }^{[1,2]}$ has underpinned the intuition of colloid scientists on these forces. The apparent successes of DLVO theory derive from its simplicity, which allows the extraction of the essential physics of the problem of lyophobic colloid stability. Its starting point is the ansatz that colloidal particle interactions (stability or coagulation of dispersions) is determined by the balance of two separate forces: double-layer repulsion and van der Waals attraction. However, the two forces are intimately coupled, and the separation of forces is an invalid approximation. ${ }^{[3]}$ This limits the applicability of the theory to low electrolyte concentrations $\left(1 \times 10^{-3}\right.$ to $\left.5 \times 10^{-2} \mathrm{M}\right)$. In particular, it loses its predictive ability for numerous colloidal phenomena that are dominated by the problem of ion specifici-

[a] Dr. S. Murgia, Dr. F. Portesani, Prof. M. Monduzzi

Dipartimento di Scienze Chimiche, Università di Cagliari - CSGI Cittadella Monserrato, S.S. 554 Bivio Sestu, 09042 Monserrato (Italy) Fax: (+39) 070-675-4388

E-mail: monduzzi@unica.it

[b] Prof. B. W. Ninham

Department of Applied Mathematics

Australian National University, Canberra 0200 (Australia) ty, or Hofmeister effects. This problem, extant for more than a century, is one for which, indeed, the original DLVO theory did not claim any competence. Moreover, the further approximations are that: 1) the solvent is a bulk continuum, unperturbed by an electrolyte; 2) no profile of surface-induced solvent order (hydration) exists at an idealized interface; and, especially, 3) no perturbation of this hydration occurs due to interaction with adsorbed hydrated ions. (The first and third assumptions remain open. The second is justified thermodynamically by the theorem that for the evaluation of free energies, it is sufficient that the distribution function, that is, the profile of order, is correct only to zeroth approximation).

Theoretical developments have treated the electrostatic double-layer interactions by a nonlinear theory of electrolytes. The second kind of (nonelectrostatic, NES) forces have been handled by the linear theory of Lifshitz that in principle deals with all many-body quantum mechanical fluctuation forces. This procedure in which one force is treated by a nonlinear theory and the others by a linear theory violates both the Gibbs adsorption equation and the gauge condition on the electromagnetic field. ${ }^{[3,4]}$ Consequently, it can be shown that specific ion effects are missed. The term "specific ion effects" then disguises the fact that there has been no encompassing theory of systems involving aqueous electrolytes except in the limit of extreme dilution (the situation has been reviewed recently ${ }^{[5]}$ ). The problem is endemic and occurs not just for colloidal interactions, but also for the Born energies of transfer, correlation free energies of electrolytes (Debye-Hückel theory), and interfacial tensions. When the defects are remedied by treating both kinds of forces at the same level in a consistent nonlinear 
theory, specific ion effects do show up, apparently predictably. ${ }^{[6]} \mathrm{Co}-$ and counterion specificity manifests itself in a variety of processes too numerous to list here. They range from bacterial growth, ${ }^{[7]}$ enzymatic activity, ${ }^{[8,9]}$ to self-assembly of surfactants, ${ }^{[10,11]}$ to hydrophobic chromatography, ${ }^{[12]}$ and the effects can usually be ordered (at least at a qualitative level) within the well-known Hofmeister series. ${ }^{[13]}$ The series is often reversed and depends on surface or substrate, and buffer type, a fact alone that implicates the NES forces.

While progress has been made by working just at the level of continuum solvent approximation, the major source of the effects is still debated. This is not surprising, for the extension of theory just discussed includes neither bulk salt and surface-induced water structure, nor effects of dissolved gas. $^{[14,15]}$

To extend DLVO theory and account for ion specificity, two parallel lines of thought have been followed, which apparently sit in apposition and opposition to each other. One assigns to ionic hydration the entire responsibility for and carriage of the ion-specific effects. ${ }^{[16,17]}$ The other insists on the fact that ions near an interface must experience a potential due to (NES) dispersion forces in concert with the classical electrostatic potential. ${ }^{[3,6]}$ Basically, the first approach ascribes ion specificity to ionic influence on water structure, and the other to relative ionic affinity to interfaces. Both effects are certainly operative. The question is which dominates? The first approach is more complicated, as hydrated ion-ion interactions or hydrated ion-surface interactions necessarily involve more, or less, overlap of hydration shells, which leads to the concept of "hard" and "soft" ions, which itself goes back to the "civilized" model of electrolytes of Stokes. ${ }^{[18]}$ Although the second focuses on surface-ion interactions via forces not included in conventional theory, the NES ionic and solvent frequency-dependent dielectric susceptibilities (as exemplified by excess polarizabilities) play a role in both theories. The (self) interaction of a bare ion with water in the presence of its neighbors and/or an interface via NES interactions ${ }^{[19-21]}$ gives rise to strong or weak hydration and the characterization of ions as kosmotropic or chaotropic. These hydrated or "dressed" ions then experience further specific NES dispersion potentials directly with an interface, and with its profile of hydration. The two apparently different approaches are consistent, and the second in fact include at least part of the first. Which effect dominates in a particular case is a matter of dispute. ${ }^{[22,23]}$ This fact renders somewhat ambiguous the attribution of experimental results to one approach (bulk effects), as opposed to the other (surface effects). An emblematic example is the following: Dialkyldimethylammonium bromide (DDAB)/water (W)/hydrocarbon mixtures show a large reverse (water-inoil) microemulsion region, ${ }^{[2-26]}$ whereas changing the DDAB counterion to sulfate (DDAS) results in a normal (oil-in-water) microemulsion. ${ }^{[10,11]}$ Titration of the DDAS/ water/hydrocarbon microemulsion with a solution of $\mathrm{NaBr}$ induces a transition back to the reverse-phase system. Surprisingly, the change in curvature occurs at a mole fraction of bromide of 0.4. ${ }^{[1]}$ Typical counterion (sulfate) concentra- tions are around $1 \mathrm{~m}$. Such a result clearly indicates a marked preference of bromide for the interface with respect to sulfate. From an electrostatic point of view this is, of course, plainly absurd. The divalent sulfate ion ought to be the clear winner in a competition for the charged surface with the univalent bromide ion. This behavior can be attributed to the stronger attractive NES interaction between bromide and the interface, as determined by its higher polarizability. Although dispersion forces must be called into question, there are no evident reasons (experimental results) to argue against the fact that preference of bromide for the charged surface could also be due to its chaotropic behavior (sulfate is a kosmotropic ion). Therefore, in such an example both proposed theories hold. (Earlier preliminary studies of coion and counterion effects in this system revealed some extraordinary changes in the phase diagrams ${ }^{[27]}$ ).

The specific problem: We recently observed that the $\mathrm{DDAB} /$ water$/ n$-decane ternary system may provide an ideal system to explore ion-specific (Hofmeister) effects. ${ }^{[28]}$ Unusually, for the present system, dissolved gas is not a problem. Salt (counterion) concentrations are on the order of molar throughout, and gas solubility drops to essentially zero at $1 \mathrm{~m}$. The effect of adding various salts on the large microemulsion region which dominates the phase diagram was investigated. The DDAB/salt molar ratio was always kept so small that, in terms of electrostatic interactions, the added salt was expected to make only a minor contribution. It was shown that such a small addition of salt did not induce significant changes in the microscopic molecular parameters, such as chemical shifts, relaxation times, and selfdiffusion coefficients measured by NMR techniques. On the other hand, dramatic (and salt-concentration-dependent) effects were ascertained at a macroscopic level: the thermodynamically stable microemulsion (at a given surfactant-to-oil ratio) became an emulsion or a clear two-phase system at much lower water content than in the absence of any salt. Similar effects were previously observed as a result of the addition of minute amounts of $\mathrm{NaBr}$ and $\mathrm{Na}_{2} \mathrm{SO}_{4}$ to the $\mathrm{DDAB} /$ water/ $n$-dodecane system. ${ }^{[29]}$ Surprisingly, the major effects could be ascribed to minute amounts of added cations, here coions, while the anion (counterion) effects seemed to be negligible.

Herein the focus is on the interactions that strongly affect the phase behavior in the microemulsion region and on the role played by the sodium cation in the stability of these positively charged interfaces. A detailed investigation was performed by ${ }^{2} \mathrm{H}$ and ${ }^{23} \mathrm{Na}$ NMR techniques on selected samples in the thermodynamically stable microemulsion and lamellar phases when small amounts of $\mathrm{NaBr}$ were added. Then, different sodium salts and bromide salts were investigated for their effects on the DDAB/W swollen lamellar phase. 


\section{Results and Discussion}

The microemulsion phase: It is known that DDAB/water $/ n$ alkane microemulsions show a bicontinuous microstructure at low water content. With changing component ratios the microstructure gradually disconnects to form water-in-oil spherical droplets, either at high water and low surfactant content or at very high oil content. ${ }^{[24,25,30,31]}$ Figure 1 shows the region of existence of the isotropic microemulsion

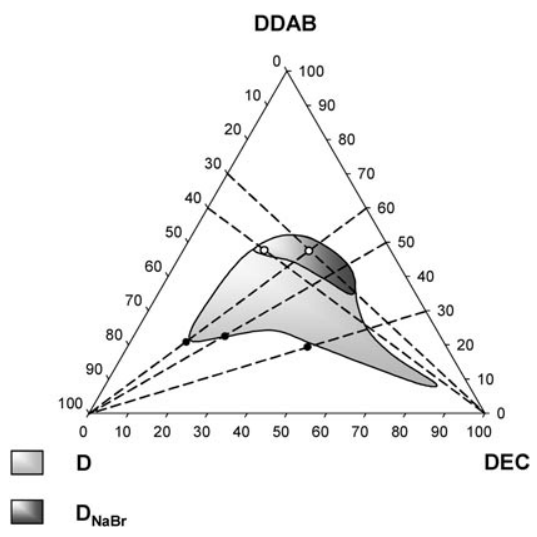

Figure 1 . The $\mathrm{DDAB} / \mathrm{D} / \mathrm{DEC}$ ternary phase diagram. $\mathrm{L}_{2}$ phase region boundaries in the presence (darkest area) and in the absence of $\mathrm{NaBr}$ are depicted along with the water dilution lines investigated by conductivity. Filled symbols indicate the percolation transition from conducting to nonconducting samples. Empty symbols indicate the composition of the two microemulsion samples investigated by NMR spectroscopy (see text). phase, in the presence and in the absence of $\mathrm{NaBr}$, in the $\mathrm{DDAB} /{ }^{2} \mathrm{H}_{2} \mathrm{O}$ (D) $/ n$-decane (DEC) ternary phase diagram, at $25^{\circ} \mathrm{C}$. The phase boundaries are slightly different from those previously reported due to the use of deuterated water. In Figure 1 the water dilution lines investigated through conductivity are also shown along with the composition of the two microemulsion samples (see below).

Independent of the presence of $\mathrm{NaBr}$, both microemulsions evolve to a water-in-oil disconnected domain with increasing water content. In the presence of $\mathrm{NaBr}$ a two-phase system forms at much lower water content than without salt (cf. Figure 1). Phase separation begins when the bicontinuous water network starts to disconnect, as indicated by a decrease in conductivity.

It seemed interesting to investigate microstructural features in the microemulsions in the proximity of phase separation by NMR measurements. Two compositions along the oil dilution lines at water/surfactant (w/s) mass ratios of 30/ 70 and 40/60 with oil contents of 32.4 ( $\mu \mathrm{e} 1$ ) and $21.4 \mathrm{wt} \%$ $(\mu \mathrm{e})$, respectively, were chosen. Table 1 lists the experimental NMR results along with some calculated parameters for $\mu \mathrm{e} 1$ and $\mu \mathrm{e} 2$ in the absence and in the presence of added $\mathrm{NaBr}$ in the aqueous phase. $\mathrm{A} D \mathrm{DAB} / \mathrm{Na}=125$ molar ratio was used. A $0.04 \mathrm{M}$ solution of $\mathrm{NaBr}$ in ${ }^{2} \mathrm{H}_{2} \mathrm{O}$ was used as reference for ${ }^{23} \mathrm{Na}$ and ${ }^{2} \mathrm{H} \mathrm{NMR}$ data.

${ }^{2} H$ NMR data: water dynamics: The ${ }^{2} \mathrm{H}$ spin-lattice $\left(R_{1}\right)$ and spin-spin $\left(R_{2}\right)$ relaxation rates show that water dynamics always occur within the extreme narrowing limit since $R_{1} \approx$ $R_{2}$. Only the reorientational correlation time becomes

Table 1. Experimental NMR data at $25^{\circ} \mathrm{C}\left(R_{1,2}=\right.$ spin-lattice (1) and spin-spin (2) relaxation rates in $\mathrm{s}^{-1} ; D=$ self-diffusion coefficient in $\left.\mathrm{m}^{2} \mathrm{~s}^{-1}\right)$ and calculated parameters $\left(\tau=\right.$ correlation times in $\mathrm{s} ; \eta_{\mathrm{app}}=$ calculated viscosity in $\mathrm{cP}$ ). Composition: $\mu \mathrm{e} 1$ (DDAB/D/DEC $\left.=47.3 / 20.3 / 32.4\right), \mu \mathrm{e} 2(\mathrm{DDAB} / \mathrm{D} /$ $\mathrm{DEC}=47.1 / 31.5 / 21.4)$. Molar ratio $\mathrm{DDAB} / \mathrm{Na}=125$.

\begin{tabular}{|c|c|c|c|c|c|}
\hline Parameter & $\mu \mathrm{e} 1$ & $\mu \mathrm{e} 2$ & $\mu \mathrm{e} 1+\mathrm{NaBr}$ & $\mu \mathrm{e} 2+\mathrm{NaBr}$ & $0.04 \mathrm{~m} \mathrm{NaBr}$ \\
\hline & \multicolumn{5}{|c|}{ Deuterated water (D) data } \\
\hline$R_{1}\left({ }^{2} \mathrm{H}\right)$ & $6.12 \pm 0.03$ & $4.56 \pm 0.01$ & $6.06 \pm 0.03$ & $4.58 \pm 0.02$ & $2.30 \pm 0.01$ \\
\hline$R_{2}\left({ }^{2} \mathrm{H}\right)$ & $6.17 \pm 0.02$ & $4.65 \pm 0.06$ & $6.03 \pm 0.04$ & $4.62 \pm 0.03$ & $2.30 \pm 0.02$ \\
\hline${ }^{\mathrm{D}} D$ & $(2.9 \pm 0.1) \times 10^{-10}$ & $(3.5 \pm 0.1) \times 10^{-10}$ & $(2.9 \pm 0.1) \times 10^{-10}$ & $(3.2 \pm 0.1) \times 10^{-10}$ & $(1.80 \pm 0.05) \times 10^{-9}$ \\
\hline $\mathrm{w} / \mathrm{s}$ & 9.78 & 15.52 & 9.78 & 15.52 & \\
\hline $\mathrm{w} / \mathrm{Na}$ & & & 1223 & 1940 & \\
\hline$\eta_{\text {app }}(\mathrm{R})^{[\mathrm{a}]}$ & 2.97 & 2.22 & 2.92 & 2.22 & 1.11 \\
\hline$\eta_{\mathrm{app}}(D)^{[\mathrm{b}]}$ & 2.37 & 1.96 & 2.37 & 2.15 & \\
\hline \multirow[t]{2}{*}{$R_{\text {cyl }}[\AA]$} & 14 & 19 & 14 & 19 & \\
\hline & \multicolumn{5}{|c|}{ Surfactant (s) data } \\
\hline$R_{1}\left({ }^{14} \mathrm{~N}\right)$ & $32.4 \pm 0.2$ & $27.6 \pm 0.2$ & $31.8 \pm 0.2$ & $27.5 \pm 0.2$ & \\
\hline$R_{2}\left({ }^{14} \mathrm{~N}\right)$ & $92.4 \pm 0.3$ & $131.8 \pm 0.2$ & $89.8 \pm 0.2$ & $127.1 \pm 0.2$ & \\
\hline${ }^{\mathrm{s}} D\left({ }^{1} \mathrm{H}\right)$ & $(1.37 \pm 0.07) \times 10^{-11}$ & $(1.51 \pm 0.08) \times 10^{-11}$ & $(1.53 \pm 0.08) \times 10^{-11}$ & $(1.48 \pm 0.07) \times 10^{-11}$ & \\
\hline$R_{2}-R_{1}$ & 60.0 & 104.2 & 58.0 & 99.6 & \\
\hline \multirow[t]{2}{*}{$\tau_{\mathrm{c}}^{\text {slow }}$} & $2.5 \times 10^{-7}$ & $4.3 \times 10^{-7}$ & $2.4 \times 10^{-7}$ & $4.2 \times 10^{-7}$ & \\
\hline & \multicolumn{5}{|c|}{$\mathrm{Na}^{+}$data } \\
\hline$R_{1}\left({ }^{23} \mathrm{Na}\right)$ & & & $35.3 \pm 0.5$ & $26.6 \pm 0.3$ & $20.65 \pm 0.08$ \\
\hline$R_{2}\left({ }^{23} \mathrm{Na}\right)$ & & & $47.8 \pm 0.7$ & $34.4 \pm 0.5$ & $20.64 \pm 0.08$ \\
\hline${ }^{\mathrm{Na}} D\left({ }^{23} \mathrm{Na}\right)$ & & & $(1.1 \pm 0.1) \times 10^{-10}$ & $(1.2 \pm 0.1) \times 10^{-10}$ & $(1.06 \pm 0.02) \times 10^{-9}$ \\
\hline$\Delta R_{\mathrm{ex}}$ & & & 0.54 & 0.43 & \\
\hline$\tau_{\mathrm{c}}^{\text {slow }}$ & & & $3.1 \times 10^{-9}$ & $4.3 \times 10^{-9}$ & $7.34 \times 10^{-12}$ \\
\hline$R_{2}-R_{1}$ & & & 12.5 & 7.8 & \\
\hline$p_{\mathrm{B}} S_{\mathrm{b}}^{2}$ & & & 0.0038 & 0.0016 & \\
\hline$R_{\mathrm{h}}[\AA]$ & & & & & 1.9 \\
\hline
\end{tabular}

[a] $1.11 \times 10^{-3}\left[\left(R_{1}+R_{2}\right) / 2\right] / 2.3$, where $\eta=1.11 \times 10^{-3} \mathrm{cP}$ is the viscosity of $\mathrm{D}$ and $2.3 \mathrm{~s}^{-1}$ is the relaxation rate of $\mathrm{D}$ in the presence of $0.04 \mathrm{M} \mathrm{NaBr}$. [b] $1.11 \times$ $10^{-3}\left[\left(6.2 \times 10^{-10}\right) / \mathrm{D} D\right]$, where ${ }^{\mathrm{D}} D=6.2 \times 10^{-10} \mathrm{~m}^{2} \mathrm{~s}^{-1}$ is the expected self-diffusion coefficient of $\mathrm{D}$ molecules that move freely in one direction. 
slower as a result of the limited amount of water in the water domain of the microemulsions. Indeed w/s molar ratios of 9.78 and 15.52 for $\mu \mathrm{e} 1$ and $\mu \mathrm{e} 2$, respectively, indicate that most water molecules are water of hydration. It has been suggested that bulk water occurs only for w/s > $15,{ }^{[32]}$ and therefore its apparent reorientational correlation time increases from about $3 \mathrm{ps}$ in pure water to $6-8 \mathrm{ps}$ in the microemulsions. This is confirmed also by the values of the water self-diffusion coefficients ${ }^{\mathrm{D}} D$. Whereas in the $0.04 \mathrm{M}$ $\mathrm{NaBr}$ solution a ${ }^{\mathrm{D}} D$ value of $1.80 \times 10^{-9} \mathrm{~m}^{2} \mathrm{~s}^{-1}$, very close to that reported for pure deuterium oxide $\left(1.87 \times 10^{-9} \mathrm{~m}^{2} \mathrm{~s}^{-1}\right)$, is measured, values around $(2.9-3.5) \times 10^{-10} \mathrm{~m}^{2} \mathrm{~s}^{-1}$ are measured in the microemulsions. This order of magnitude is typical of bicontinuous microemulsions in which interconnected water domains exist and water self-diffusion is almost free in one direction. In such case ${ }^{\mathrm{D}} D={ }^{\mathrm{D}} D^{\circ} / 3 \approx 6.2 \times 10^{-10} \mathrm{~m}^{2} \mathrm{~s}^{-1}$ would be expected.$^{[33]}$ However, as observed in other bicontinuous microemulsions, the measured ${ }^{\mathrm{D}} D$ value is smaller due to the slowly diffusing water molecules in the hydration shell of polar interfaces. Nevertheless, almost free diffusion in one direction can be assumed. No restricted diffusion was ascertained, since the measured self-diffusion coefficients do not depend on the observation time $\Delta-\delta / 3$ (experiments were performed with $\Delta$ varying in the range $40-200 \mathrm{~ms}$ ).

Water molecules of hydration cause both an increase in the relaxation rates and a decrease in the self-diffusion coefficients. It can be inferred that the apparent viscosity of the water domain increases with respect to the bulk. Indeed the reorientational correlation time $\tau_{\mathrm{c}}$ is linearly dependent on viscosity $\eta$ [Eq. (1)], where $R_{\mathrm{h}}$ is the hydrodynamic radius, $k$ the Boltzmann constant, and $T$ the absolute temperature. The self-diffusion coefficient shows an inverse dependence on viscosity [Eq. (2)].

$\tau_{\mathrm{c}}=\frac{3 \pi \eta R_{\mathrm{h}}^{3}}{k T}$

$D=\frac{k T}{6 \pi \eta R_{\mathrm{h}}}$

Taking into consideration these two equations, a two- to threefold increase of the microviscosity of the water domain can be predicted. The apparent viscosities $\eta_{\text {app }}$ calculated from relaxation data and from diffusion measurements are reported in Table 1. Therefore, it can be assumed that in $\mu \mathrm{e} 1$ with $\mathrm{w} / \mathrm{s}=9.78$ and in $\mu \mathrm{e} 2$ with $\mathrm{w} / \mathrm{s}=15.52$ the viscosity increases by a factor of 1.9 and 2.5 , respectively. These data are quite consistent with the dimensions of the water conduits that can be calculated for the approximation of a cylindrical shape ${ }^{[32]}$ according to Equation (3), where $R_{\text {cyl }}$ is the radius of the cylindrical conduit, $v_{\mathrm{w}}$ the volume of a water molecule $\left(30 \AA^{3}\right), a_{\mathrm{s}}$ the area per polar head group $\left(68 \AA^{2}\right),{ }^{[34,35]}$ and $R_{\mathrm{s}}$ is a contribution due to the polar head group (here $R_{\mathrm{s}}=5 \AA$ was used ${ }^{[32]}$ ). These data will be further considered in the analysis of the effects of $\mathrm{Na}^{+}$ions.
$R_{\mathrm{cyl}}=\frac{2 v_{\mathrm{w}}}{a_{\mathrm{s}}} \frac{w}{s}+R_{\mathrm{s}}$

A general comment arises from these data. Water parameters are not affected by the presence of sodium. The main effect is due to the different amounts of water in the two microemulsions. This influences the local microviscosity of the water network and determines the different dynamics.

${ }^{14} N$ and ${ }^{1} H$ NMR data: surfactant dynamics: Several previous papers ${ }^{[26,31,36]}$ have demonstrated the sensitivity of ${ }^{14} \mathrm{~N}$ NMR relaxation as a technique to investigate percolation and microstructural transitions in DDAB systems. Indeed the favorable location of nitrogen in the DDAB polar head group and the intrinsic properties of the ${ }^{14} \mathrm{~N}$ nucleus (spin $I=1)$ makes it a readily available and sensitive probe to monitor slow motions and fluctuations up to the microsecond timescale. The two-step model approach ${ }^{[37]}$ was used to show that, from the difference between the $R_{2}$ and $R_{1}$ relaxation rates, the correlation time for the slow motion can be obtained from Equation (4), where $S_{\mathrm{b}}$ is the order parameter related to the bound site, $\chi$ is the quadrupolar coupling constant of the ${ }^{14} \mathrm{~N}$ nucleus, and the various $J(\omega)$ are defined as a function of the slow correlation time related to the "bound" species $\tau_{\mathrm{c}}^{\text {slow }}$ [Eq. (5)].

$$
\begin{aligned}
& R_{2}-R_{1}=\left[\frac{9 \pi^{2}}{40}\right]\left(S_{\mathrm{b}} \chi\right)^{2}\left[J^{\mathrm{S}}(0)+J^{\mathrm{S}}\left(\omega_{\mathrm{N}}\right)-2 J^{\mathrm{S}}\left(2 \omega_{\mathrm{N}}\right)\right] \\
& \approx\left[\frac{9 \pi^{2}}{40}\right]\left(S_{\mathrm{b}} \chi\right)^{2} J^{\mathrm{S}}(0) \\
& J(\omega)=\frac{2 \tau_{\mathrm{c}}^{\text {slow }}}{1+\omega^{2}\left(\tau_{\mathrm{c}}^{\text {slow }}\right)^{2}}
\end{aligned}
$$

In Equation (4), the product $\chi S_{\mathrm{b}}$ can be obtained from the ${ }^{14} \mathrm{~N}$ quadrupolar splittings measured in the $\mathrm{L}_{\alpha}$ phase, by assuming the fraction of the observed nucleus in the bound state $p_{\mathrm{b}}=1$ [Eq. (6)].

$\Delta v_{\mathrm{q}}=\frac{3}{4} p_{\mathrm{B}} S_{\mathrm{b}} \chi$

The value of $\chi S_{\mathrm{b}}$ here used is $10.4 \mathrm{kHz}$, as previously observed. ${ }^{[26]}$

The difference $\Delta R=R_{2}-R_{1}$ in Equation (4) is mainly affected by slow geometry-dependent motions, since the contribution associated with local fast motions, which are independent of the interfacial geometry, is subtracted. These slow motions involve surfactant lateral diffusion along the curved interface and reorientation of the interface itself. The calculated $\tau_{\mathrm{c}}^{\text {slow }}$ are very similar to those determined previously in percolating DDAB/water/DEC microemulsions. ${ }^{[31]}$ A slight dependence on the composition is observed for $\tau_{\mathrm{c}}^{\text {slow }}$. It increases with increasing w/s. More remarkably, it increases with decreasing oil volume fraction from $\varphi_{\mathrm{o}}=0.40$ in $\mu \mathrm{e} 1$ to 0.28 in $\mu \mathrm{e} 2$. The state of bicontinuous microemulsions is further confirmed by the self-diffusion coefficients deter- 
mined by ${ }^{1} \mathrm{H}$ NMR PGSTE experiments. Minimal differences were observed in the two microemulsions.

In summary, the dynamics of the surfactant interface, exactly as for the water dynamics, is not affected by the presence of $\mathrm{Na}^{+}$and $\mathrm{Br}^{-}$ions in the polar domain.

${ }^{23} \mathrm{Na} N \mathrm{NM}$ data: $\mathrm{Na}^{+}$ion dynamics: The ${ }^{23} \mathrm{Na}$ nucleus has a spin quantum number of $I=3 / 2$ and the treatment of relaxation data of systems outside the extreme narrowing limit, as in the present case $\left(R_{1} \neq R_{2}\right)$, is less straightforward than for spin $I=1$, as for the ${ }^{14} \mathrm{~N}$ nucleus. Moreover, here $\mathrm{Na}^{+}$ions are not the counterions of a charged interface, so that strong binding cannot be expected. What must be picked up from ${ }^{23} \mathrm{Na}$ NMR relaxation and diffusion is essentially a weak coion interaction. Both $R_{1}$ and $R_{2}$ relaxation decays were suitably fitted by single exponential functions. Here, two simplified equations that allow estimates of the slow correlation time $\tau_{\mathrm{c}}^{\text {slow }}$ and the product $p_{\mathrm{b}} S_{\mathrm{b}}^{2}$ between the $\mathrm{Na}^{+}$bound fraction $p_{\mathrm{b}}$ and the order parameter $S_{\mathrm{b}}$ were used. A detailed description of the theory underlying the ${ }^{23} \mathrm{Na} \mathrm{NMR}$ multiexponential relaxation in the slow motion regime is reported elsewhere. ${ }^{[30,32,38]}$

Assume that ${ }^{23} \mathrm{Na}$ NMR relaxation measured in the bulk reference $0.04 \mathrm{M} \mathrm{NaBr}$ solution $\left(R_{1} \approx R_{2}=20.65 \mathrm{~s}^{-1}\right)$ represents the free-ion relaxation contribution $R^{\text {free }}$, from which a correlation time $\tau_{\mathrm{c}}=7.34 \mathrm{ps}$ can be calculated by introducing $\chi=665.8 \mathrm{kHz}^{[38]}$ and using Equation (7), where the asymmetry parameter $\eta^{2} / 3$ was neglected, as is usually done in symmetric environments. Now assume a simple two-site model. We can define as "bound ions" those located near to a slowly moving interface, close enough to experience an interaction. To get information on the dynamics an estimate of the slow correlation time of the "bound" species can be obtained by calculating the excess relaxation rates $R_{1 \mathrm{ex}}$ and $R_{2 \mathrm{ex}}$ from the Equations (8).

$R_{1}=R_{2}=R^{\text {free }}=\left(\frac{2 \pi^{2}}{5}\right) \chi^{2} \tau_{\mathrm{c}}$

$R_{1 \mathrm{ex}}=R_{1}-R^{\mathrm{free}}$ and $R_{2 \mathrm{ex}}=R_{2}-R^{\mathrm{free}}$

These represent a measure of the involvement of $\mathrm{Na}^{+}$ ions in the aggregation phenomena. Then all the unknown parameters that occur in the relaxation equations can be neglected if the ratio between the excess relaxation rates $R_{\text {1ex }}$ and $R_{2 \mathrm{ex}}$ is considered [Eq. (9)].

$\Delta R_{\mathrm{ex}}=\frac{R_{1 \mathrm{ex}}}{R_{2 \mathrm{ex}}}=\frac{[0.2 J(\omega)+0.8 J(2 \omega)]}{[0.3 J(0)+0.5 J(\omega)+0.2 J(2 \omega)]}$

By using Equations (4), (8), and (9) correlation times of 3.1 and $4.3 \mathrm{~ns}$ were estimated for $\mu \mathrm{e} 1$ and $\mu \mathrm{e} 2$, respectively, in the presence of $\mathrm{NaBr}$. These correlation times are consistent with the observation, at our experimental field strength of $7 \mathrm{~T}(1 / \omega \approx 4 \mathrm{~ns})$, of a single exponential decay for both $R_{1}$ and $R_{2}$. Now introducing the calculated $\tau_{\mathrm{c}}^{\text {slow }}$ values into Equation $(10),{ }^{[30,32,38]}$ and assuming again $\chi=665.8 \mathrm{kHz}$ gives the parameters $p_{\mathrm{b}} S_{\mathrm{b}}^{2}=0.0038$ for $\mu \mathrm{e} 1$ and 0.0016 for $\mu$ 2. Order parameters are usually quite small in the symmetric and isotropic environments that occur in a microemulsion. For instance, a value of $p_{\mathrm{b}} S_{\mathrm{b}}^{2}=0.028$ was found for $\mathrm{Na}^{+}$counterions in a sodium dodecylsulfate micellar solution (5 wt \% SDS in water) ${ }^{[38]}$ Considering that in this relation the order parameter appears as a square, this is a striking result. It can be inferred that a significant fraction of $\mathrm{Na}^{+}$ions closely experiences the presence of the DDAB cationic interface.

$R_{2}-R_{1}=p_{\mathrm{B}} S^{2}\left(\frac{2 \pi^{2}}{5}\right) \chi^{2}[0.3 J(0)+0.3 J(\omega)-0.6 J(2 \omega)]$

It might be argued that $\mathrm{Na}^{+}$ions are forced to reside close to the cationic interface as a result of the size of the water domain (cf. cylinder radii of 14 and $19 \AA$ ) and the absence of real bulk water molecules $(\mathrm{w} / \mathrm{s} \leq 15)$. We do not believe this to be the case. This assertion can be demonstrated as follows. A hydrodynamic radius $R_{\mathrm{h}}$ of $1.9 \AA$ (the Pauling ionic radius is $0.95 \AA^{[39]}$ ) can be calculated from Equation (2) by using the sodium self-diffusion coefficient ${ }^{\mathrm{Na}} D=$ $1.06 \times 10^{-9} \mathrm{~m}^{2} \mathrm{~s}^{-1}$. Such a hydrodynamic radius must be interpreted as the radius of a sphere constituted by the ion and its tightly bound water molecules that move with the diffusing ion. The $R_{\mathrm{h}}$ value of sodium calculated here is in excellent agreement with those already reported $(1.8 \AA) .{ }^{[40]}$ The values of $D_{\mathrm{Na}}$ decrease by one order of magnitude in the microemulsions. However, this, as in the case of water diffusion, is likely to be due to the increase in microviscosity. Indeed, if $R_{\mathrm{h}}=1.9 \AA$ and the average apparent viscosity of $\mathrm{D}$ reported in Table 1 are introduced into Equation (2), and considering also that diffusion may occur in one direction only, values of ${ }^{\mathrm{Na}} D=1.67 \times 10^{-10} \mathrm{~m}^{2} \mathrm{~s}^{-1}$ for $\mu \mathrm{e} 1$ and $1.52 \times$ $10^{-10} \mathrm{~m}^{2} \mathrm{~s}^{-1}$ for $\mu \mathrm{e} 2$ can be predicted. These data are quite close to those experimentally measured.

The main conclusion is clear: the interaction between $\mathrm{Na}^{+}$ions and the DDAB cationic interface exists, as proven particularly by ${ }^{23} \mathrm{Na}$ NMR relaxation. This is reasonable since it is known that quadrupolar nuclei are very sensitive to slow motion of the bound species. On the contrary, selfdiffusion coefficients are mainly determined by the hydrodynamic radius, the dimension and the shape of the domain over which the observed species can move, and finally by the viscosity of the medium. The main objection to this conclusion resides in the absence of real bulk water. Therefore, $\mathrm{Na}^{+}$ions may be forced to be located close to interface, although the number of water molecules per sodium ion is very high (see w/Na in Table 1$)$.

To go further, the effect of sodium salt addition was investigated in the lamellar phase of the DDAB/D binary system.

The lamellar phase: The phase diagram of the DDAB/D binary system has been previously investigated ${ }^{[36,41,42]}$ by optical microscopy and ${ }^{2} \mathrm{H}$ and ${ }^{14} \mathrm{~N}$ NMR spectroscopy. Two types of lamellar phases occur. These are a swollen lamellar phase $\mathrm{L}_{\alpha 1}$ that coexists with liposomes and forms at high 
water content, and a compact lamellar phase $\mathrm{L}_{\alpha 2}$ that forms only for $\mathrm{DDAB} / \mathrm{D}$ mass ratio higher than $80 / 20$. In the range of composition between 25 and $80 \mathrm{wt} \%$ DDAB the two lamellar phases coexist over a wide two-phase region. A critical upper consolute point was identified at about $80^{\circ} \mathrm{C}$ in the presence of deuterated water. ${ }^{[36]}$ This critical point decreases to $72{ }^{\circ} \mathrm{C}$ when normal water is used. ${ }^{[42]}$

Several samples were prepared with the same molar ratio $\mathrm{DDAB} / \mathrm{Na}=125$. Figure 2 a shows the ${ }^{23} \mathrm{Na} \mathrm{NMR}$ spectrum

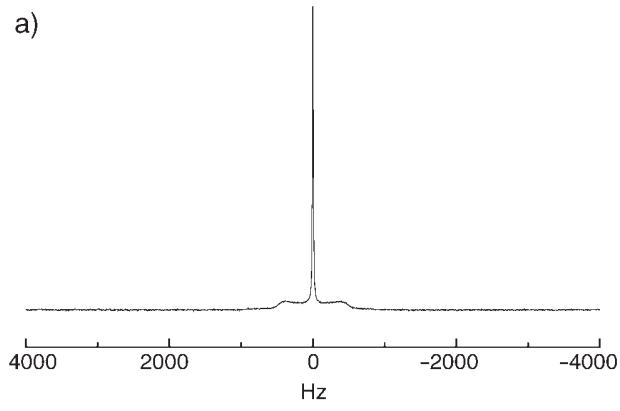

b)

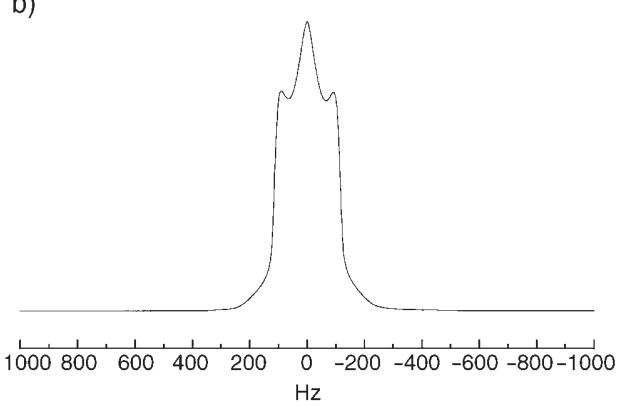

Figure 2. a) ${ }^{23} \mathrm{Na}$ and b) ${ }^{2} \mathrm{H}$ NMR spectra of a $\mathrm{DDAB} / \mathrm{D}=18 / 82 \mathrm{~L}_{\alpha 1}$ sample $\left(\mathrm{DDAB} / \mathrm{Na}^{+}=125\right)$.

obtained with a mass ratio of $\mathrm{DDAB} / \mathrm{D}=18 / 82$. Here both the central ${ }^{23} \mathrm{Na} \mathrm{NMR}$ signal and two satellites can clearly be seen, with a quadrupolar splitting of $\Delta v_{\mathrm{q}} \approx 400 \mathrm{~Hz}$. Recalling that the quadrupolar splitting [see Eqs.(6) and (11)] for the lamellar phase is strictly related to the number of nuclei located within a short distance $(5-6 \AA)$ of the anisotropically oriented interface, ${ }^{[32]}$ this is again a striking result. In this case $w / s \approx 105$. This means that there are more than 13000 molecules of water per sodium ion. In the swollen lamellar phase a spacing of about $110 \AA$ has been ascertained ${ }^{[42]}$ moreover, the lamellar packing coexists with a liposome microstructure. The occurrence of significant liposome organization is demonstrated by the ${ }^{2} \mathrm{H}$ NMR spectra, where, besides the doublet due to the quadrupolar splitting of oriented D molecules, an isotropic signal is always seen. Figure $2 \mathrm{~b}$ shows the ${ }^{2} \mathrm{H}$ NMR spectrum, which confirms the coexistence of the two microstructures. Preliminary relaxation and self-diffusion measurements indicate the occurrence of two different environments (lamellar and liposome) without exchange between them of both water and sodium species. Although the amount of bulk water is quite large in this $L_{\alpha 1}$ phase, a significant amount of sodium resides suffi- ciently close to the DDAB cationic interface to experience the lamellar anisotropic orientation in the magnetic field.

From previous investigations on DDAB with different counterions, the preferential binding of bromide with respect to other counterions has been demonstrated. ${ }^{[11,27,43]}$ Consequently, one possibility is that, on addition of $\mathrm{NaBr}$, the binding of bromide counterions increases and, for electrostatic reasons, $\mathrm{Na}^{+}$ions also come close to the interface. To test this hypothesis, a number of other sodium salts were examined. Table 2 shows the ${ }^{23} \mathrm{Na}$ NMR quadrupolar split-

Table 2. ${ }^{23} \mathrm{Na}$ NMR splittings at $25^{\circ} \mathrm{C}$ for different sodium salts in the $\mathrm{L}_{\alpha 1}$ phase of a sample with mass ratio $\mathrm{DDAB} / \mathrm{D}=18 / 82$. A molar ratio of $\mathrm{DDAB} / \mathrm{Na}=125$ was used. The reproducibility is within $\pm 5 \%$.

\begin{tabular}{ll}
\hline Sodium salt & $\Delta v_{\mathrm{q}}[\mathrm{Hz}]$ \\
\hline $\mathrm{NaBr}$ & 410 \\
$\mathrm{NaF}$ & 390 \\
$\mathrm{NaCl}$ & 408 \\
$\mathrm{NaClO}_{4}$ & 400 \\
$\mathrm{NaNO}_{3}$ & 405 \\
$\mathrm{Na}_{2} \mathrm{SO}_{4}$ & 400 \\
\hline
\end{tabular}

tings measured in the presence of $\mathrm{NaF}, \mathrm{NaCl}, \mathrm{NaNO}_{3}$, $\mathrm{NaClO}_{4}$, and $\mathrm{Na}_{2} \mathrm{SO}_{4}$ : very similar results were obtained. This rules out an assignment of the effect to electrostatics alone. It can be concluded that the anion is not important in determining the attraction of the sodium cations to the DDAB cationic interface.

However, at this stage, an obvious question arises. Is this a specific property of the $\mathrm{Na}^{+}$ion? Looking at the past literature an answer can be found: it is not necessary to have a purely electrostatic interaction to attract a charged ion close to an oriented interface. This was the case of 1-monoctanoin/water lamellar phase in the presence of $\mathrm{NaCl}$ and $\mathrm{CsCl}$ (also in the presence of $\mathrm{NaCl}$ and $\mathrm{LiCl}$ to evaluate competitive binding),${ }^{[44-46]}$ for which ${ }^{23} \mathrm{Na} \Delta v_{\mathrm{q}}$ in the range $4-12 \mathrm{kHz}$ and ${ }^{133} \mathrm{Cs} \Delta v_{\mathrm{q}}$ in the range $70-400 \mathrm{~Hz}$ were measured. Since the surfactant is a nonionic monoglyceride, it was suggested that a major contribution to the splitting must arise from distortions of the ionic hydration shell for ions entering the oriented water layer (usually two water layers with a total thickness of $4 \AA$ are considered). Later it was demonstrated that when the chloride lyotropic series of monovalent cations $\mathrm{Li}^{+}, \mathrm{Na}^{+}, \mathrm{K}^{+}, \mathrm{Rb}^{+}$, and $\mathrm{Cs}^{+}$is added to an anionic surfactant/water system different phase transitions were observed. ${ }^{[47]}$ This behavior was mainly ascribed to the different hydration and different hydrated radii of the cations (see Table 3). The radii reported in Table 3 correspond to those first reported by Conway, ${ }^{[48]}$ which differ from those calculated from the Stokes equation [e.g., Eq. (2)].

In this context it seemed interesting to ascertain on our $\mathrm{DDAB} / \mathrm{W} \mathrm{L}_{\alpha 1}$ phase the effect of the bromide lyotropic series of the same monovalent cations. Using the same $\mathrm{DDAB} /$ cation molar ratio and the same procedures as above, the various ${ }^{7} \mathrm{Li},{ }^{23} \mathrm{Na},{ }^{39} \mathrm{~K},{ }^{87} \mathrm{Rb}$, and ${ }^{133} \mathrm{Cs} \mathrm{NMR}$ spectra were recorded. These nuclei have a spin quantum number of $I=3 / 2$, with the exception of ${ }^{133} \mathrm{Cs}(I=7 / 2)$. Very different $\Delta v_{\mathrm{q}}$ were measured for the different nuclei. Only 
Table 3. Quadrupole moments of different monovalent cations and observed quadrupolar splittings of the corresponding bromide salts added $(\mathrm{DDAB} /$ cation molar ratio $=125)$ to the DDAB/W $(18 / 82) \mathrm{L}_{\alpha}$ phase.

\begin{tabular}{llclllc}
\hline Nucleus & $I$ & $\begin{array}{c}|\mathrm{Q}| \\
{\left[\mathrm{fm}^{2}\right]}\end{array}$ & $F$ & $\begin{array}{l}\text { Hydration } \\
\text { number }\end{array}$ & $\begin{array}{l}\text { Radius }^{[a]} \\
{[\AA]}\end{array}$ & $\begin{array}{c}\Delta v_{\mathrm{q}}^{\text {obs }} \\
{[\mathrm{Hz}]}\end{array}$ \\
\hline${ }^{7} \mathrm{Li}$ & $3 / 2$ & 4.01 & 2.00 & 7.4 & 3.8 & $10 \pm 0.5$ \\
${ }^{23} \mathrm{Na}$ & $3 / 2$ & 10.4 & 5.20 & 6.5 & 3.6 & $400 \pm 4$ \\
${ }^{39} \mathrm{~K}$ & $3 / 2$ & 5.85 & 2.92 & 5.1 & 3.3 & - \\
${ }^{87} \mathrm{Rb}$ & $3 / 2$ & 13.35 & 6.675 & 4.7 & 3.3 & $2800 \pm 10$ \\
${ }^{133} \mathrm{Cs}$ & $7 / 2$ & 0.343 & 0.024 & 4.3 & 3.3 & $14 \pm 0.5$ \\
\hline
\end{tabular}

[a] Data from ref. [50].

${ }^{39} \mathrm{~K}$ NMR spectra did not show any evidence of possible quadrupolar splittings, probably due to the low sensitivity of the nucleus itself accompanied by an unfavorably short spin-spin relaxation time. Data are reported in Table 3 along with some nuclear parameters.

If in the case of the chloride lyotropic series and the anionic surfactant some competitive effects specific to counterion binding were evident, ${ }^{[47]}$ then a direct comparison among the $\Delta v_{\mathrm{q}}^{\text {obs }}$ values cannot be sustained. Indeed, Equation (6) can be rewritten in its more general form [Eq. (11)], ${ }^{[4,49]}$ where $\theta_{\mathrm{LD}}$ is the angle between the magnetic field and the director (the interface symmetry axis), $\theta_{\mathrm{DM} i}$ the angle between the director and the $z$ axis of the coordinate system at nuclear site $i$ (the term in square brackets represents the order parameter of site $i$ ), $p_{i}$ the fraction of ions at site $i$, and $v_{\mathrm{Q}}$ the quadrupolar coupling constant. Many unknown parameters occur in Equation (11). If the nuclear parameters that are known and do not depend on the site $i$ are grouped in the term $F=3 Q /[2 I(2 I-1)]$, where $Q$ is the nuclear quadrupole moment (see Table 3 ), the following decreasing order of the quadrupolar splittings might be predicted: ${ }^{87} \mathrm{Rb}>{ }^{23} \mathrm{Na}>{ }^{39} \mathrm{~K}>{ }^{7} \mathrm{Li}>{ }^{133} \mathrm{Cs}$. The $\Delta v_{\mathrm{q}}^{\text {obs }}$ values reported in Table 3 confirm this trend only for the ${ }^{87} \mathrm{Rb}$ and ${ }^{23} \mathrm{Na}$ nuclei. However, this is not very surprising. The different hydration, hydrated radius, and polarization of the various cations may be important factors. On the other hand, besides several recent papers, ${ }^{[5,20,50]}$ it has been demonstrated that even coion adsorption may occur at weakly charged membranes. ${ }^{[51]}$ This was suggested to result in a number of nonlinear phenomena due to nonuniformities in the fixedcharge distribution (local defects).

$\Delta v_{\mathrm{q}}=\mid\left(3 \cos ^{2} \theta_{\mathrm{LD}}-1\right) \sum_{i} p_{i} v_{\mathrm{Q}}\left[0.5\left(3 \cos ^{2} \theta_{\mathrm{DM} i}-1\right)\right]$

\section{Discussion and Conclusion}

To recap the main findings:

1) Very small additions of $\mathrm{NaBr}$ to a microemulsion formed by the system of cationic surfactant didodecyldimethylammonium bromide, deuterated water, and decane cause an impressive shrinkage of the region of existence of this thermodynamically stable phase.
2) ${ }^{14} \mathrm{~N},{ }^{2} \mathrm{H}$, and ${ }^{23} \mathrm{Na}$ NMR relaxation and self-diffusion investigations of DDAB, D, and $\mathrm{Na}^{+}$ion indicate that water and surfactant dynamics are not altered by the presence of the added salt, and suggest that $\mathrm{Na}^{+}$ions closely approach the positively charged interface.

3) This point was thoroughly investigated in the lamellar phase of the DDAB/D binary system. The NMR spectrum of this sample shows a quadrupolar splitting as a result of the interaction of the ${ }^{23} \mathrm{Na}$ quadrupole moment with an anisotropically oriented interface. This interaction is well recognized as a short-range property. Therefore, without doubt, the inevitable conclusion is that $\mathrm{Na}^{+}$ ions reside in the proximity of the cationic interface (within 5-6 A). Similar conclusions can be drawn for the other monovalent cations, particularly for $\mathrm{Rb}^{+}$ions. This occurs despite the huge amount of bulk water in the lamellar phase.

4) A number of samples prepared using different sodium salts demonstrate that attraction of the $\mathrm{Na}^{+}$ion to the interface is not anion specific.

How can such scarcely credible results be explained? A simple effect of electrostatic interactions alone can be discarded a priori. Calling into play "hydration" forces could hardly be a solution to the problem. Indeed, if we think in terms of hydrogen-bond weakening/enhancing ions, the small, weakly polarizable, kosmotropic sodium ions should be repelled from the interface. By so doing they can better decrease the total free energy of the system in bulk water. Moreover, this condition should be enhanced in our system by the high charge density of the interface, which induces a high ionic concentration at the interface ${ }^{[43]}$ and a consequent smaller dielectric constant than in the bulk. Therefore, sodium ions should also be repelled toward the bulk via image forces.

On the contrary, some things seem to fall into place if (NES) dispersion forces are taken into account. Depending on the ion and on the interface the nonelectrostatic quantum-mechanical Lifshitz dispersion potentials can be either repulsive or attractive. Although less significant at low electrolyte concentration, this potential can dominate when electrostatic forces are screened. In our lamellar system the DDAB concentration is $0.5 \mathrm{M}$ (well outside the expected validity of DLVO theory) and a Debye length of less than $5 \AA$ can be estimated. This is exactly the distance that the various cations need to "feel" the interfacial anisotropic gradient field that allows quadrupolar splitting in the NMR spectrum. The circle is not quite closed. The membrane rigidity in the "counterion-only" case of ionic charged interfaces is peculiar and lies outside the intuition of normal doublelayer theory (e.g., a reverse phase of water/surfactant in oil would collapse in a continuum solvent model and cannot exist without hydration interactions between head groups). ${ }^{[52-55]}$.

Therefore, even without invoking the NES or dispersion forces that are certainly operative, the introduction of a small amount of salt, as in our case, could give rise to a sin- 
gular perturbation in membrane rigidity. Where does that bring us?

The salt-free case: One possible clue to events lies in consideration of what is known about the microstructure of the DDAB/water/alkane system without salt. The microstructure has been extensively explored both experimentally and theoretically. ${ }^{[24-26,31,34,35]}$ Essentially it comes about, and can be predicted, from a combination of just two factors. These are the (effectively) constant curvature of the surfactant interface set by a balance of intermolecular forces due to head-group repulsion, and hydrocarbon interactions. The former are counterion-specific (principally hydration), and the latter oil-specific, corresponding to the degree of oil penetration. The second constraint is global packing, set by volume fractions. Most remarkable of all, even the critical exponents for the antipercolation threshold at constant water-to-surfactant ratio emerge precisely. ${ }^{[31]}$ The same considerations appear to be quite general, and apply to systems as apparently different as the $\mathrm{CuAOT} /$ water/isooctane system. ${ }^{[56]}$ However, the successes of the simple model of disordered connected cylinders (DOC) $)^{[34,35]}$ are not the whole story. For example, the microstructure of the DDAB/ water/tetradecane system, which has no connection to the oil corner in the ternary phase diagram, ${ }^{[26]}$ is very different. Its explanation is very much more subtle and requires a complicated transition of a monolayer to a connected random (nonbirefringent) bilayer structure. ${ }^{[57]}$ This is one clue, as the interface with a nonpenetrating oil such as tetradecane is much more fluid than either the DDAB system with low alkanes or the CuAOT system. The transition from connected conduits to disconnected droplets within the $\mathrm{L}_{2}$ phase is a consequence of global packing. But once the global conditions that allow packing of such spheres are violated, the subsequent breaking of the microemulsion, with increasing water content, to form a spontaneous emulsion (of unknown structure) must be much more complicated. Whatever its complicated structure is, it does involve multilamellar bilayers or cylinders, again apparently nonbirefringent. Hence, a refolding of monolayers to form bilayers must occur, just as for the tetradecane microemulsion system. This is reasonable for high water content at which the emulsion forms as the monolayer is confronted with an increasing amount of bulk water, with a corresponding decrease in bending modulus.

The salt-containing case: If we compare the case with a small amount of salt with the salt-free case, the boundary of the emulsion region occurs at a water content that has nothing to do with global packing, even though the microstructure is exactly as predicted by the DOC model within the microemulsion region. As we cross this boundary, a twophase emulsion region forms, as for the salt-free case.

To explore this further, a biphasic sample of composition $\mathrm{DDAB} / \mathrm{D}(\mathrm{Na}) / \mathrm{DEC}=15 / 36 / 49$ was left to stand at $25^{\circ} \mathrm{C}$ for one week to obtain gravitational separation of an upper isotropic phase and a lower turbid phase. The extremely low conductivity $\left(<1 \mu \mathrm{S} \mathrm{cm}^{-1}\right)$ and a water self-diffusion coefficient of $2.1 \times 10^{-11} \mathrm{~m}^{2} \mathrm{~s}^{-1}$ confirm the existence of a water-inoil microemulsion in the upper phase. Conversely, optical microscopy (see Figure 3) strongly suggests an emulsionlike

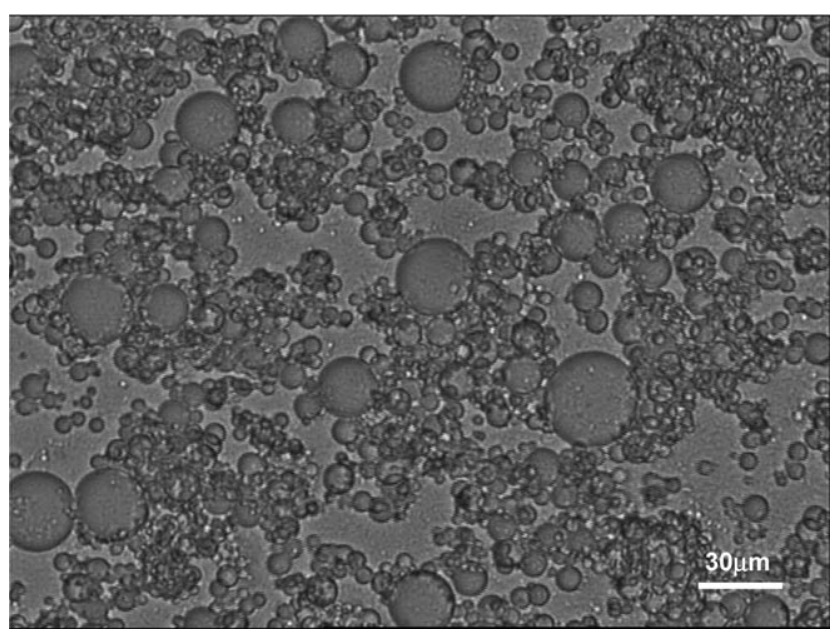

Figure 3. Light micrograph of the turbid lower phase of the DDAB/D $\mathrm{D}_{\mathrm{Na}}$ $\mathrm{DEC}=15 / 36 / 49$ sample recovered after gravitational separation.

constitution of the lower phase. Moreover, the observation of an intense ${ }^{23} \mathrm{Na}$ NMR signal in the turbid phase and a very small signal in the isotropic solution implies that the emulsion contains most of the $\mathrm{Na}^{+}$ions. Therefore, most $\mathrm{Na}^{+}$ions have been expelled from the microemulsion phase during the microstructural transition from a water continuous network to water-in-oil droplets. The emulsion contains the bulk of the ions and has a much larger area of surfactant than the upper microemulsion phase. A reasonable conclusion is then that the adsorbed sodium ions, at sufficient water content, congregate and provide a sufficient source of defects in the surfactant monolayer region that join conduits to weaken the monolayer bending modulus. This allows a preferred refolding of the conduits into bilayers and an emulsion.

If this is correct then it leads on to the idea of nanocompartmentalization in such complicated systems that has already been foreshadowed in biological systems.

While the possibility that ions affect the structure of water has been recently considered to be negligible, ${ }^{[5-61]}$ ion-water interactions certainly do exist, and the preference of a charged ion for media of higher dielectric constant is not in doubt. Nevertheless, here the delicate energy competition at a positively charged interface (that determines the ion distribution near the surface) is won by NES dispersion forces of quantum origin.

Considering the vast number of biological and nonbiological systems in which charged soft interfaces are involved, the implications are large. And the possibility that such phenomena occur also in anionic or nonionic interfaces appears much more than a remote. ${ }^{[4-46]}$ There is much theoretical work $^{[19-21,50,62]}$ from which this kind of phenomenon involv- 
ing apparently counterintuitive coion adsorption at charged interfaces can be inferred, but this study apparently provides direct confirmation. Inclusion of dispersion forces into a modified DLVO theory may well not be the only answer to all phenomena outside the reach of standard theories, but it appears to be a significant step in the right direction.

\section{Materials and Methods}

Didodecyldimethylammonium bromide (DDAB) and decane (DEC), both with $98 \%$ grade of purity, were purchased from Fluka. Deuterated water (D), with a purity of $99.9 \%$ was obtained from Cambridge Isotopes Laboratories, Inc. $\mathrm{NaBr}$ (purity $99.5 \%$ ), $\mathrm{NaCl}$ (purity $99.5 \%$ ), $\mathrm{NaF}$ (purity $99 \%$ ), $\mathrm{NaNO}_{3}$ (purity $99 \%$ ), and $\mathrm{Na}_{2} \mathrm{SO}_{4}$ (purity $99 \%$ ), were purchased from Sigma-Aldrich; KBr (purity $99.5 \%$ ), RbBr (purity 99\%), and $\mathrm{CsCl}$ (purity $99.5 \%$ ) were obtained from Fluka; $\mathrm{NaClO}_{4}$ (purity $99 \%$ ) was purchased from Acros Organics, and $\mathrm{LiBr}$ (purity 99\%) from Riedel-De-Haen. All reagents were used as received.

The region of existence of the reversed microemulsion region in the $\mathrm{DDAB} / \mathrm{D} / \mathrm{DEC}$ phase diagram with and without the addition of $\mathrm{NaBr}$ was explored by visual inspection or, where necessary, by measuring the absorbance at $400 \mathrm{~nm}$ of the samples with a Varian Cary $50 \mathrm{UV} / \mathrm{Vis}$ spectrophotometer. $\mathrm{NaBr}$ was added such that in each point of the phase diagram the $\mathrm{DDAB} / \mathrm{NaBr}$ molar ratio was 125 . (We emphasize: there is only one perturbing coion per 125 dimethylammonium cationic head groups, while counterion concentrations are essentially unperturbed). Phase boundaries are reported with an accuracy of $\pm 2 \mathrm{wt} \%$.

Conductivity measurements were performed along three different water dilution lines (DDAB/DEC mass ratios of 60/40, 50/50, and 30/70) using a Microprocessor Conductivity Meter from WTW.

Microemulsion samples for NMR analysis were prepared by mixing DDAB, DEC, and D or, as appropriate, a solution of $\mathrm{NaBr}$ in deuterated water $\left(D_{\mathrm{N}_{2}}\right)$ in a glass tube by using a vortex mixer. The binary samples in the lamellar $\mathrm{L}_{\alpha 1}$ phase (see below) were prepared by mixing DDAB and $\mathrm{D}$ or $\mathrm{D}_{\mathrm{Na}}$ in a glass tube by means of a vortex mixer. All samples were stored at $25^{\circ} \mathrm{C}$ for at least one week prior to any NMR analyses. ${ }^{2} \mathrm{H},{ }^{7} \mathrm{Li},{ }^{23} \mathrm{Na},{ }^{39} \mathrm{~K},{ }^{87} \mathrm{Rb}$, and ${ }^{133} \mathrm{Cs}$ NMR experiments were performed on a Bruker Avance $300 \mathrm{MHz}(7.05 \mathrm{~T})$ spectrometer at respective operating frequencies of 46.072, 116.642, 79.390, 14.005, 98.204, and $39.365 \mathrm{MHz}$. The spectrometer was equipped with a Bruker field-gradient probe DIFF30 that can reach field gradients of up to $12.0 \mathrm{Tm}^{-1}$. A standard variable-temperature control unit with an accuracy of $0.5^{\circ} \mathrm{C}$ was used. Spin-lattice $\left(R_{1}\right)$ and spin-spin $\left(R_{2}\right)$ relaxation rates were obtained by means of the usual inversion recovery (180- $\tau-90$-acquisition) and CPMG (90- $\tau-180-2 \tau-180-4 \tau-\ldots$-acquisition) acquisition sequences, respectively. The spin-lattice relaxation rates $R_{1}$ and the spin-spin relaxation rates $R_{2}$ were obtained by a three- [for $R_{1}$, Eq. (12)] and two-parameter [for $R_{2}$, Eq. (13)] nonlinear fitting of the partially relaxed NMR signal intensities obtained at 14-18 different $\tau$ values. The error in the measurements, as judged by repeated measurements, is reported as standard deviation in the Tables.

$I(\tau)=A-B \exp \left(-\tau R_{1}\right)$

$I(\tau)_{\text {echo }}=C \exp \left(-\tau R_{2}\right)$

Self-diffusion coefficients were obtained by means of the pulse field gradient stimulated echo (PGSTE) sequence by varying the gradient strength $g$ while keeping the gradient pulse length $\delta$ and the gradient pulse intervals $\Delta$ constant. Data were fitted to the modified StejskalTanner equation for the PGSTE sequence [Eq. (14)] $]^{[63,64]}$
$I\left(\mathrm{~g}, \delta, \Delta, \tau_{1}, T\right)=I_{0} \exp \left[\left(-2 \frac{\tau_{1}}{T_{2}}\right)-\left(\frac{T}{T_{1}}\right)\right] \exp \left[-D \gamma^{2} g^{2} \delta^{2}\left(\Delta-\frac{\delta}{3}\right)\right]$

where $I$ and $I_{0}$ are the echo intensity in the presence and in the absence of the applied field gradient, respectively, $\gamma$ is the gyromagnetic ratio of the investigated nucleus, $\tau_{1}$ and $T$ are the constant times between the first and the second, and the second and the third $90^{\circ}$ pulse, $T_{1}$ and $T_{2}$ the spin-lattice and spin-spin relaxation times, and $D$ is the self-diffusion coefficient.

Repeated measurements up to six months after sample preparation showed no difference in the NMR parameters, while variations in the appearance of quadrupolar powder spectra (not in the quadrupolar splittings) were sometimes detected, most likely because of the equilibration of the lamellar microdomains along the preferential orientation axis. ${ }^{[6]}$

All the NMR measurements were repeated at least five times. Errors are reported in terms of standard deviation.

Optical micrographs were obtained through an optical microscope Zeiss Axioplan 2.

All experimental measurements as well as the phase diagram determination were carried out at $25^{\circ} \mathrm{C}$.

\section{Acknowledgements}

MIUR-Prin 40\% (Italy), and Consorzio Sistemi Grande Interfase (CSGI, Italy) are acknowledged for financial support. B.W.N. thanks MIUR (Italy) for a visiting professor position within the "Rientro dei cervelli" project 2003.

[1] B. V. Derjaguin and L. Landau, Acta Physicochim. URSS 1941, 14, $633-662$.

[2] J. Verwey, J. T. G. Overbeek, Theory of the Stability of Lyophobic Colloids, Elsevier, 1948.

[3] B. W. Ninham, V. Yaminsky, Langmuir 1997, 13, 2097-2108.

[4] W. Kunz, P. Lo Nostro, B. W. Ninham, Curr. Opin. Colloid Interface Sci. 2004, 9, 1-18.

[5] Curr. Opinion Colloid Interface Sci., Vol. 9 (Eds.: E. W. Kaler, K. Holmberg, B. H. Robinson), Elsevier, Amsterdam, 2004

[6] B. W. Ninham, Adv. Colloid Interface Sci. 1999, 1, 1-17.

[7] P. Lo Nostro, A. Lo Nostro, B. W. Ninham, G. Pesavento, L. Fratoni, P. Baglioni, Curr. Opin. Colloid Interface Sci. 2004, 9, 97-101.

[8] H.-K. Kim, E. Tuite, B. Norden, B. W. Ninham, Eur. Phys. J. A 2001, 4, 411-417.

[9] M. C. Pinna, A. Salis, M. Monduzzi, B. W. Ninham, J. Phys. Chem. B 2005, 109, 5406-5408.

[10] M. Nyden, O. Soderman, Langmuir 1995, 11, 1537-1545.

[11] M. Nyden, O. Soderman, P. Hansson, Langmuir 2001, 17, 67946803.

[12] K. D. Collins, Proc. Natl. Acad. Sci. USA 1995, 92, 5553-5557.

[13] K. D. Collins, M. W. Washabaugh, Quart. Rev. Biophys. 1985, 18, $323-422$.

[14] M. E. Karaman, B. W. Ninham, R. M. Pashley, J. Phys. Chem. 1996, $100,15503-15507$.

[15] M. Alfridsson, B. W. Ninham, S. Wall, Langmuir 2000, 16, 1008710091.

[16] M. Manciu, E. Ruckenstein, Adv. Colloid Interface Sci. 2003, 105, 63-101.

[17] E. Ruckenstein, M. Manciu, Adv. Colloid Interface Sci. 2003, 105, $177-200$.

[18] R. A. Robinson, R. H. Stokes, Electrolyte Solutions, 2nd revised ed., Butterworths, London, 1965.

[19] M. Bostrom, B. W. Ninham, Langmuir 2004, 20, 7569-7574.

[20] M. Bostrom, B. W. Ninham, J. Phys. Chem. B 2004, 108, $12593-$ 12595. 
[21] M. Bostrom, B. W. Ninham, Biophys. Chem. 2005, 114, 95-101.

[22] M. Bostrom, F. W. Tavares, S. Finet, F. Skouri-Panet, A. Tardieu, B. W. Ninham, Biophys. Chem. 2005, 117, 115-122.

[23] A. Salis, M. C. Pinna, D. Bilanikova, M. Monduzzi, P. Lo Nostro, B. W. Ninham, J. Phys. Chem. B 2006, 110, 2949.

[24] F. D. Blum, S. Pickup, B. W. Ninham, S. J. Chen, D. F. Evans, J. Phys. Chem. 1985, 89, 711.

[25] S. J. Chen, D. F. Evans, B. W. Ninham, D. J. Mitchell, F. D. Blum, S. Pickup, J. Phys. Chem. 1986, 90, 842-847.

[26] M. Monduzzi, F. Caboi, F. Larché, U. Olsson, Langmuir 1997, 13, 2184-2190.

[27] V. Chen, D. F. Evans, B. W. Ninham, J. Phys. Chem. 1987, 91, 18231826.

[28] S. Murgia, M. Monduzzi, B. W. Ninham, Curr. Opin. Colloid Interface Sci. 2004, 9, 102-106.

[29] R. Skurtveit, U. Olsson, J. Phys. Chem. 1991, 95, 5353-5358.

[30] M. Monduzzi, F. Caboi, C. Moriconi, Colloids Surf. 1997, 129-130, $327-338$.

[31] M. Monduzzi, S. Mele, J. Phys. Chem. B 2001, 105, 12579-12582.

[32] I. Furo, B. Halle, P.-O. Quist, T. C. Wong, J. Phys. Chem. 1990, 94, 2600-2613.

[33] D. F. Evans, H. Wennerstrom, The Colloidal Domain, VCH Publishers, New York, 1994.

[34] S. T. Hyde, J. Phys. Chem. 1989, 93, 1458-1464.

[35] S. T. Hyde, B. W. Ninham, T. Zemb, J. Phys. Chem. 1989, 93, 14641471.

[36] F. Caboi, M. Monduzzi, Langmuir 1996, 12, 3548-3556.

[37] H. Wennerstrom, B. Lindman, O. Soderman, T. Drakenberg, J. B. Rosenholm, J. Am. Chem. Soc. 1979, 101, 6860-6864.

[38] A. Ceglie, G. Colafemmina, M. D. Monica, L. Burlamacchi, M. Monduzzi, J. Colloid Interface Sci. 1991, 146, 363-371.

[39] J. E. Huheey, E. A. Keiter, R. L. Keiter in Inorganic Chemistry: Principles of Structure and Reactivity, 4th ed., HarperCollins, New York, 1993

[40] M. Y. Kiriukhin, K. D. Collins, Biophys. Chem. 2002, 99, 155-168.

[41] K. Fontell, A. Ceglie, B. Lindman, B. W. Ninham, Acta Chem. Scand. 1986, A40, 247.

[42] T. Zemb, D. Gazeau, M. Dubois, T. Gulik-Krzywicki, Europhys. Lett. 1993, 21, 759.

[43] R. M. Pashley, P. M. McGuiggan, B. W. Ninham, D. F. Evans, J. Brady, J. Phys. Chem. 1986, 90, 1637-1642.

[44] G. Lindblom, B. Lindman, G. J. T. Tiddy, J. Am. Chem. Soc. 1978, 100, 2299-2303.
[45] H. Wennerstrom, N.-O. Persson, G. Lindblom, B. Lindman, G. J. T. Tiddy, J. Magn. Reson. Zeitschrift wurde erst 1997 geründet! 1978, 30, $133-136$.

[46] N.-O. Persson, G. Lindblom, J. Phys. Chem. 1979, 83, 3015-3019.

[47] A. Sein, J. B. F. N. Engberts, Langmuir 1995, 11, 455-465.

[48] B. E. Conway, Ionic Hydration in Chemistry and Biophysics, Elsevier, Amsterdam, 1981.

[49] G. Lindblom in Advances in Lipids Methodology-Three (Ed.: W. W. Christie), The Oily Press, Dundee, 1996, Chap. 5.

[50] M. Bostrom, D. R. M. Williams, B. W. Ninham, Langmuir 2001, 17, 4475-4478.

[51] V. M. Aguilella, J. A. Manzanares, J. Pellicer, Langmuir 1993, 9 , 550-554.

[52] A. Fogden, D. J. Mitchell, B. W. Ninham, Langmuir 1990, 6, 159162.

[53] A. Fogden, B. W. Ninham, Langmuir 1991, 7, 590-595.

[54] A. Fogden, J. Daicic, D. J. Mitchell, B. W. Ninham, Physica 1996, 234, 167-188.

[55] A. Fogden, B. W. Ninham, Adv. Colloid Interface Sci. 1999, 83, 85110.

[56] P. Andre, B. W. Ninham, P. Pileni, New J. Chem. 2001, 25, 563-571.

[57] M. Olla, A. Semmler, M. Monduzzi, S. T. Hyde, J. Phys. Chem. B 2004, 108, $12833-12841$.

[58] A. W. Omta, M. F. Kropman, S. Woutersen, J. Chem. Phys. 2003, $119,12457-12461$.

[59] A. W. Omta, M. F. Kropman, S. Woutersen, H. J. Bakker, Science 2003, 301, 347-349.

[60] L.-Å. Naslund, D. C. Edwards, P. Wernet, U. Bergmann, H. Ogasawara, L. G. M. Pettersson, S. Myneni, A. Nilsson, J. Phys. Chem. A 2005, 109, 5995-6002.

[61] L.-Å. Naslund, J. Luning, Y. Ufuktepe, H. Ogasawara, P. Wernet, U. Bergmann, L. G. M. Pettersson, A. Nilsson, J. Phys. Chem. B 2005, 109, 13835-13839.

[62] M. Bostrom, D. R. M. Williams, B. W. Ninham, Phys. Rev. Lett. 2001, 87, 168103/168101-168104

[63] E. O. Stejskal, J. E. Tanner, J. Chem. Phys. 1965, 42, 288-292.

[64] L. Ambrosone, S. Murgia, G. Cinelli, M. Monduzzi, A. Ceglie, J. Phys. Chem. B 2004, 108, 18472-18478.

[65] P. L. Hubbard, K. M. McGrath, P. T. Callaghan, Langmuir 2005, 21, 4340-4346.

Received: January 18, 2006 Published online: July 18, 2006 